\title{
Aspectos cinemáticos e neuromusculares relacionados com o desempenho da saída do bloco na natação
}

\author{
CDD. 20.ed. 621.811 \\ 796.073 \\ 797.2
}

\author{
Daniele DETANICO* \\ Stephanie Iara HEIDORN ${ }^{*}$ \\ Gustavo Ricardo SCHÜTZ* \\ Saray Giovana dos SANTOS
}

*Universidade Federal

de Santa Catarina

\begin{abstract}
Resumo
0 objetivo deste estudo foi relacionar o desempenho (distância e tempo de voo) na saída de bloco com variáveis cinemáticas e neuromusculares. Participaram 10 nadadores do sexo masculino $(26,4 \pm 6,6$ anos, $76,4 \pm 6,6 \mathrm{~kg}, 1,81 \pm 0,07 \mathrm{~m}$ de estatura). Utilizou-se uma câmera filmadora (30 Hz) para a aquisição das variáveis cinemáticas, sendo analisadas: distância de voo, tempo de voo, altura máxima do quadril, ângulos de saída de bloco e entrada na água. Para a obtenção da potência muscular foi utilizado o "Squat Jump" realizado sobre uma plataforma de força. Foram obtidas as seguintes variáveis: altura do salto, potência e pico de velocidade. Utilizou-se estatística descritiva e correlação de Pearson para relacionar as variáveis neuromusculares e cinemáticas com o desempenho na saída de bloco, utilizando nível de significância de 5\%. Encontrou-se correlação significativa do ângulo de saída com a distância $(r=$ $-0,59)$ e com o tempo de voo $(r=0,88)$, além da altura máxima com o tempo de voo $(r=0,59)$. Não foi observada correlação significativa da distância e do tempo de voo com as variáveis neuromusculares ( $p$ $>0,05$ ). Com base nos resultados pode-se concluir que o desempenho na saída de bloco da natação foi relacionado com o ângulo de saída de bloco e com a altura máxima do quadril na fase aérea. A potência muscular não interferiu no desempenho nos nadadores investigados.
\end{abstract}

UnIteRMos: Natação; Força muscular; Cinemática.

\section{Introdução}

Em esportes cujo tempo é o grande adversário, como na natação (HALJAND, 1998), se reconhece que a eliminação de uma técnica incorreta deva ser um objetivo importante, e para isso devem se analisar as possíveis causas desse defeito para que se compreenda que métodos deverão ser utilizados para sua correção. A natação competitiva chegou a um estágio no qual a vitória e quebra de recordes dependem de diferenças pequenas de segundos e centésimos de segundos, tornando o aperfeiçoamento da técnica um fator fundamental na busca por um melhor desempenho (Araújo, Pereira, Gatti, Freitas, Jacomel, Roesler \& Vilas-Boas, 2010).

Para a análise da técnica na natação, assim como em outras modalidades, opta-se pela divisão do evento a ser estudado em partes menores, em seus fundamentos. Assim, uma melhora de tempo em um determinado fundamento, como na saída, pode representar a diferença necessária para a vitória, corroborando com GuimarÁES e HAY (1985), em que o nadador precisa não somente nadar rápido, mas também executar rapidamente a saída e as viradas (se houver).

O desempenho da saída, mensurado pelo tempo gasto pelo nadador percorrer a distância de $15 \mathrm{~m}$ iniciais de uma prova pode representar de $0,8 \%$ a $26,1 \%$ do tempo total de uma prova, de acordo com a distância da mesma (Cossor \& MASON, 2001). Desta forma, este se torna essencial em provas de velocidade, podendo garantir uma vantagem significativa para o nadador (BLANKSBY, SIMPSON, ELLIOT \& McElroY, 1998), com uma importância decrescente a medida que a distância da prova aumenta (Cossor \& MASON, 2001), mas sem deixar de ser importante (Hubert, 2005). Considerando esse aspecto, LeWIS 
(1980) aponta duas vantagens sendo a primeira o fator psicológico de estar na frente na prova e a segunda o fato de estar nadando em águas mais calmas, o que pode aumentar a eficiência da braçada.

Por envolver fases desenvolvidas tanto fora da água quando submersas, a saída de bloco também pode ser decomposta, com sua descriçãao e análise, segundo Haljand (1998), constituído por um sistema de movimentos com fases delimitadas de ações claras e determinadas. Assim, a saída é dividida, de acordo com Ruschel, Araújo, Pereira e Roesler (2007), em duas subseçôes: a primeira, aérea e anterior à entrada do nadador na água; e a segunda, na qual o nadador permanece submerso e avança para o início do nado. Estas subseções são analisadas por componentes temporais (tempo de bloco, de voo, submerso e de nado) ou por componentes de distância (entrada, submersa e nado) (Blanksby, Nicholson \& Elliot, 2002; Daly, Malone, Smith, Vanlandewijck \& Steadward, 2001).

Para uma saída eficiente, RusCHEL et al. (2007) e TAKeda, Ichikawa, Takagi e Tsubakimoto (2009) apontam ser necessário inicialmente, uma combinação dos movimentos realizados no bloco de partida com os de projeção para a água, obtendo distâncias e ângulos ideais, que influenciará positivamente as etapas posteriores da saída, ou seja, na fase submersa e inicial do nado. A definição destes valores ideais dos movimentos realizado no bloco tem sido objeto de diversos estudos (BurketT, Mellifont \& Mason, 2010; Miller, Allen \& Pein, 2003; Seifert, Vantorre \& Chollet, 2007; Vantorre, Seifert, FerNANDES, Vilas-Boas \& Chollet, 2010a; Welcher, HinRichs \& GEORGE, 2008), com a mensuração de variáveis biomecânicas e apontando suas contribuições para o desempenho da fase inicial (área),

\section{Métodos}

\section{Sujeitos do estudo}

Participaram deste estudo 10 nadadores meiofundistas do sexo masculino, a partir dos seguintes critérios de inclusão: a) realizar treinos de natação pelo menos quatro vezes na semana; $b$ ) não possuir nenhum tipo de lesão; c) ter 18 anos ou mais; d) realizar a saída de agarre; e) participar de competições no mínimo de nível estadual. Os nadadores apresentaram média de idade de 26,4 $\pm 6,6$ anos, massa corporal de 76,4 $\pm 6,6 \mathrm{~kg}, 1,81 \pm 0,07 \mathrm{~m}$ de estatura e tempo de prática de 7,2 $\pm 3,1$ anos. quantificado pela distância alcançada pelo nadador (distância de voo) e o tempo gasto em sua execução (tempo de voo) (COSSOR \& MASON, 2001; SEIFERT, Vantorre, Lemaitre, Chollet, Toussaint \& VilasBoas, 2010; Vantorre, Seifert, Fernandes, VilasBOAS \& CHOLlet, 2010b). Assim, pode-se apontar que nadadores que possuem uma saída eficiente, que compreende a fase de desprendimento do bloco, fase aérea e de entrada na água, tendem a apresentar maior distância de voo do que os demais, acarretando numa menor distância a ser percorrida dentro da água.

Além dos aspectos biomecânicos da técnica, o aprimoramento das qualidades físicas também é de extrema importância quando se busca a melhora do desempenho geral (GARRIDO, Marinho, ReIs, VAN Den Tillaar, Costa, Silva \& Marques, 2010) e especificamente da saída da natação (SEIFERT et al., 2010; WEST, Owen, Cunningham, CoOK \& Kilduff, 2010). Neste último, as principais capacidades físicas intervenientes são dependentes de aspectos neuromusculares, como a potência muscular dos membros inferiores (BOCALINI, Andrade, Uezu, Santos \& Nakamoto, 2007; Breed $\&$ Young, 2003; SeIFerT et al., 2010).

Apesar de a potência muscular apresentar considerável importância, poucos e recentes estudos foram encontrados que relacionassem essa variável com o desempenho das açóes iniciais realizadas no bloco de partida. Desse modo, verificar o grau de importância dessa variável no desempenho dos nadadores torna-se extremamente importante. Além disso, uma técnica perfeita no bloco de saída é parte fundamental do desempenho final da prova. Assim, este estudo teve como objetivo relacionar o desempenho (distância e tempo de voo) realizado pelos nadadores no bloco de saída com variáveis cinemáticas e neuromusculares.

\section{Instrumentos e procedimentos}

Antes de iniciarem os procedimentos para a coleta de dados, os atletas que participaram do estudo foram esclarecidos sobre os objetivos e os métodos da pesquisa, para então assinarem o Termo de Consentimento Livre e Esclarecido. O presente estudo foi aprovado pelo Comitê de Ética em Pesquisas com Seres Humanos da Universidade local (processo 809/2010).

Inicialmente foi realizada a aquisição de imagens para a análise cinemática e, após um intervalo de no mínimo 24 horas, a avaliação da potência muscular. 


\section{Protocolo da análise cinemática}

Para aquisição das variáveis cinemáticas foi utilizada uma câmera filmadora (Sony ${ }^{\circledR}$ modelo DSC-P32, $30 \mathrm{~Hz}$ ), posicionada a $3 \mathrm{~m}$ de distância do bloco de saída e a uma altura $1,20 \mathrm{~m}$. Para a calibração do espaço foram utilizadas duas hastes verticais com altura de $1,99 \mathrm{~m}$ e distando entre si 4,50 m.

Os nadadores realizaram um aquecimento prévio e na sequência uma saída de bloco. Após essa execução, os nadadores secaram-se, foi feita a marcação dos pontos anatômicos (acrômio, epicôndilo lateral do úmero, epicôndilo lateral da ulna, trocânter maior do fêmur, epicôndilo lateral da fíbula, maléolo lateral e cabeça do segundo metatarso) e então cada sujeito realizou uma saída do bloco com a técnica de agarre. Optou-se em realizar somente uma repetição, de acordo com o destacado por Hubert (2005), no qual o número deve ser o menor possível, já que pode haver uma tendência de queda de desempenho e falta motivação por parte dos nadadores. Com isto, esperava-se que uma única execução representasse o máximo desempenho do sujeito.

Após a captura, as imagens foram transferidas para um computador e os dados brutos das coordenadas dos marcadores transformados em coordenadas globais bidimensionais (2D) através do método de Transformação Linear Direta (DLT), processadas por meio do "software" SkillSpector ${ }^{\circledR}$, versão 1.2.5. A partir da análise cinemática foram obtidas as seguintes variáveis: a) distância de voo $\left(\mathrm{d}_{\text {voo }}\right)$ : alcance horizontal atingido pelos atletas após a saída, considerando o ponto do bloco de partida até o primeiro contato das mãos com a água; $b)$ tempo de voo $\left(\mathrm{t}_{\text {voo }}\right)$ : diferença entre os instantes de perda de contato com o bloco e o primeiro contato das mãos com a água; c) altura máxima $\left(\mathrm{H}_{\text {máx }}\right)$ : considerada como a maior altura atingida pela articulação do quadril durante a fase de voo em relação a linha da água.

Posteriormente foi feita a análise dos ângulos de saída de bloco e entrada na água por meio do "software" gráfico Corel Draw X3 ${ }^{\circledR}$ versão 13. Para a obtenção destes ângulos foi utilizado o método citado por HUBERT (2005), onde: o ângulo de saída do bloco $\left(\hat{A}_{\text {sai }}\right)$ é o ângulo entre o segmento de reta formado pelas referências no corpo do nadador (utilizando-se os pontos anatômicos do acrômio e maléolo lateral) e o plano horizontal; e o ângulo de entrada na água $\left(\hat{\mathrm{A}}_{\text {ent }}\right)$ é o ângulo observado no instante em que a mão do nadador toca a água, formado pelo segmento de reta (epicôndilo lateral da ulna e trocânter maior do fêmur) e o plano horizontal.

\section{Protocolo de avaliação da potência muscular}

A potência muscular dos nadadores foi avaliada a partir do desempenho obtido no salto vertical denominado "Squat Jump" (SJ). O protocolo do SJ consiste em partir de uma posição estática com o ângulo do joelho em aproximadamente $90^{\circ}$, com o tronco o mais vertical possível e as mãos na cintura, sendo que o atleta realiza o salto sem que haja um contra-movimento.Utilizou-se uma plataforma de força portátil, do tipo piezoelétrica (Kistler $^{\circledR}$, Quattro Jump, 9290AD, Winterthur, Switzerland) que mensura a força de reação do solo (FRS) no componente vertical. As informações adquiridas foram transmitidas via cabo a um computador na frequência de $500 \mathrm{~Hz}$ (Ache Dias, Dal Pupo, Reis, Borges, Santos, Moro \& Borges Junior, 2011). Foram realizados três SJ, sendo determinadas as seguintes variáveis: a) altura do salto $\left(S J_{H}\right)$ : foi calculada usando o "software" Quattro Jump por meio do método da dupla integração da força, onde inicialmente obteve-se a aceleração dividindo-se os valores da FRS pela massa corporal dos sujeitos. A partir da aceleração obteve-se a curva de velocidade, a qual foi novamente integrada para obter o deslocamento do centro de massa em cada instante do movimento, sendo o maior deslocamento vertical considerado a altura de salto; b) potência $\left(\mathrm{SJ}_{\mathrm{Pot}}\right)$ : obtida a partir da multiplicação da FRS pela velocidade na fase concêntrica do salto, sendo considerado para análise o valor médio da curva (analisou-se os valores absolutos e normalizados pela massa corporal); c) pico de velocidade $\left(\mathrm{SJ}_{\text {Pico vel }}\right)$ : maior velocidade obtida após a perda de contato dos pés com a plataforma, obtida pelo "software" Quattro Jump (Dal Pupo, Detanico \& Santos, 2011).

\section{Análise estatística}

Para a apresentação dos resultados foi utilizada estatística descritiva (média, desvio-padrão e coeficiente de variação). Através do teste de ShapiroWilk foi verificada a normalidade dos dados e então, para correlacionar as variáveis cinemáticas e neuromusculares dos nadadores com o desempenho na saída do bloco determinado pela $d_{\text {voo }}$ e $t_{\text {voo }}$, foi utilizada a correlação linear de Pearson, com nível de significância de 5\%. 


\section{Resultados}

Na TABELA 1 são apresentados os valores observados para as variáveis cinemáticas e neuromusculares dos nadadores investigados.

Na TABELA 2 estão apresentadas as correlações entre a $d_{\text {voo }}$ e o $t_{\text {voo }}$ com as demais variáveis cinemáticas e com os índices neuromusculares. Pode-se observar que houve correlação significativa

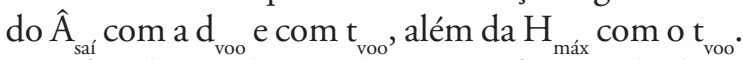
Não foi observada correlação significativa da $\mathrm{d}_{\text {voo }} \mathrm{e}$ do $t_{\text {voo }}$ com as variáveis neuromusculares.

TABELA 1- Valores descritivos das variáveis cinemáticas e neuromusculares durante a saída de bloco.

Média \pm desvio padrão
(Coeficiente de variação).

\begin{tabular}{lclc}
\hline & Variáveis cinemáticas & \multicolumn{1}{c}{ Variáveis neuromusculares } \\
\hline $\mathrm{d}_{\text {voo }}(\mathrm{m})$ & $3,30 \pm 0,16(4,5 \%)$ & $\mathrm{SJ}_{\text {Pot }}(\mathrm{W})$ & $1648,6 \pm 254,7(15,5 \%)$ \\
$\mathrm{t}_{\text {voo }}(\mathrm{s})$ & $0,40 \pm 0,05(12,6 \%)$ & $\mathrm{SJ}_{\mathrm{Pot}}\left(\mathrm{W} \cdot \mathrm{kg}^{-1}\right)$ & $21,6 \pm 3,0(13,8 \%)$ \\
$\hat{\mathrm{A}}_{\text {sai }}\left({ }^{\circ}\right)$ & $20,0 \pm 6,3(31,6 \%)$ & $\mathrm{SJ}_{\mathrm{H}}(\mathrm{cm})$ & $43,4 \pm 4,8(11,1 \%)$ \\
$\hat{\mathrm{A}}_{\text {ent }}\left({ }^{\circ}\right)$ & $39,1 \pm 10,3(26,4 \%)$ & $\mathrm{SJ}_{\text {Pico vel }}\left(\mathrm{m} \cdot \mathrm{s}^{-1}\right)$ & $2,63 \pm 0,20(7,1 \%)$ \\
$\mathrm{H}_{\text {max }}(\mathrm{m})$ & $1,40 \pm 0,12(8,6 \%)$ & & \\
\hline
\end{tabular}

TABELA 2 - Correlação entre a $\mathrm{d}_{\mathrm{voo}}$ e o $\mathrm{t}_{\mathrm{voo}}$ e as demais variáveis cinemáticas e neuromusculares.

\begin{tabular}{llcccc}
\hline & & $\mathbf{D}_{\text {voo }}(\mathbf{m})$ & $\mathbf{p}$ & $\mathbf{t}_{\mathrm{voo}}(\mathbf{s})$ & $\mathbf{p}$ \\
\hline Variáveis cinemáticas & $\hat{\mathrm{A}}_{\mathrm{sat}}(\mathrm{o})$ & $-0,59^{*}$ & 0,04 & $0,88^{* *}$ & 0,001 \\
& $\mathrm{~A}_{\mathrm{ent}}(\mathrm{o})$ & $-0,52$ & 0,12 & 0,26 & 0,47 \\
& $\mathrm{H}_{\text {max }}(\mathrm{m})$ & $-0,41$ & 0,23 & $0,59^{*}$ & 0,03 \\
Variáveis neuromusculares & $\mathrm{SJ}_{\text {Pot }}(\mathrm{W})$ & 0,51 & 0,09 & $-0,19$ & 0,59 \\
& $\mathrm{SJ}_{\mathrm{Pot}}\left(\mathrm{W} \cdot \mathrm{kg}^{-1}\right)$ & 0,49 & 0,15 & $-0,39$ & 0,27 \\
& $\mathrm{SJ}_{\mathrm{H}}(\mathrm{cm})$ & 0,47 & 0,17 & $-0,30$ & 0,41 \\
& $\mathrm{SJ}_{\text {Pico vel }}\left(\mathrm{m} . \mathrm{s}^{-1}\right)$ & 0,45 & 0,19 & $-0,30$ & 0,40 \\
\hline
\end{tabular}

\section{Discussão}

Tendo em vista o objetivo deste trabalho, em relacionar os indicadores de desempenho da saída do bloco $\left(\mathrm{d}_{\mathrm{voo}}\right.$ e $\left.\mathrm{t}_{\mathrm{voo}}\right)$ com variáveis cinemáticas e neuromusculares, o principal achado deste trabalho foi a correlação significativa entre estes indicadores e o $\hat{A}_{\text {sai }}$. Isso significa que menores $\hat{A}_{\text {sai }}$ tendem a gerar maiores $\mathrm{d}_{\mathrm{voo}}$, e possivelmente quanto menor o $\hat{\mathrm{A}}_{\mathrm{sai}}$ menor o t $\mathrm{voo}^{\circ}$.

Neste sentido, é possível sugerir que o nadador que realiza a saída de bloco com ângulos menores em relação ao plano horizontal, obterá uma melhor trajetória e, consequentemente, obterá maior distância e menor tempo de voo antes de tocar a água. Segundo Maglischo (2003), a obtenção de um ângulo ideal entre corpo do nadador e o plano horizontal no momento da saída permite ao atleta alcançar uma trajetória em arco e uma entrada correta na água. Além disso, o ângulo de saída de bloco é determinante de outras variáveis, como ângulo de entrada na água, o tempo e o alcance total (incluindo a fase submersa) e a velocidade de nado (Vaghetti, Berneira \& Roesler, 2010).

Em relação ao $\hat{\mathrm{A}}_{\text {saí }}$, outras investigações (Jorgić, Puletić, Stanković, Okičić, Bubanj \& Bubanj, 2010; Vaghetti, Berneira \& Roesler, 2010) encontraram valores angulares superiores aos reportados neste estudo, de $33,7^{\circ}$ e $35,7^{\circ}$, respectivamente, utilizando a saída de agarre. Por outro lado, Miller, Allen e Pein (2003) reportaram ângulo de saída de $14,8^{\circ}$, inferior aos encontrados no presente estudo. Vale ressaltar que o ângulo de saída apresentou elevada dispersão, indicando que a amostra se mostrou heterogênea nesta variável $(31,6 \%)$. Esta alta variabilidade também pode 
ser observada nos estudos citados anteriormente, com valores que variaram de 14,8 a $35,7^{\circ}$, que possivelmente pode ser provocada pela diferença na utilização de alguns pontos anatômicos para a definição do ângulo de saída.

Outra correlação significativa encontrada foi entre o $\mathrm{t}_{\text {voo }}$ e a $\mathrm{H}_{\text {máx }}$. Quanto menor altura o nadador atingir na saída de bloco, menor será o tempo que ele permanecerá no ar. Desta forma, reportando a visualização da saída no nadador como um lançamento de projétil, buscar maiores alturas resultaria em maiores tempos de voo e perda no desempenho final. Poder-se-ia pensar que obter uma altura mínima resultaria num desempenho ótimo, entretanto, é nesta fase de maior altura alcançado pelo nadador, que ele deve se preparar para a entrada na água. De acordo com VAGHetTI, Berneira e Roesler (2010), durante essa fase o quadril deve estar flexionado, os joelhos estendidos e a cabeça por entre os braços com os cotovelos estendidos, de modo que o nadador possa atingir a água em um bom ângulo com a horizontal, fazendo com que todo seu corpo entre em uma abertura feita pelas mãos. Deve haver então, tempo hábil para estes ajustes corporais e execução da tarefa.

Com relação ao $\hat{A}_{\text {ent }}$, os valores obtidos foram semelhantes aos observados em outros estudos (Cipolli, 2005; Mclean, Holthe, Vint, Beckett \& Hinrichs, 2000; Miller, Allen \& Pein, 2003) que encontraram ângulos entre 39,0 e 40,0. VILASBoas, Cruz, Sousa, ConceiçÃo e Carvalho (2001) e JorGić et al. (2010) encontraram ângulos de entrada na água de $28,6^{\circ}$ e $33,3^{\circ}$, respectivamente, inferiores aos achados deste estudo. Em outra investigação, VAGHetti, Berneira e Roesler (2010) observaram valores angulares de $48,7^{\circ}$, superiores aos deste estudo. A amostra analisada também apresentou uma considerável dispersão para esta variável.

Para Maglischo (2003), o ângulo de entrada na água influencia diretamente no tempo de deslize do nadador após a saída, considerando valores entre 30 e $40^{\circ}$ como ideais. Os atletas estudados apresentaram uma média próxima ao limite superior, mas com uma considerável dispersão (26,4\%). Para realmente influenciar positivamente nas fases seguintes de deslize e nado, esta variável parece ser algo a ser aprimorado por alguns dos nadadores.

Um fator que pode interferir na altura máxima e consequentemente no ângulo de entrada na água é a técnica de saída de bloco. De acordo com TAKEDA e Nomura (2006), na saída de agarre (opção realizada pelos sujeitos desta pesquisa) a velocidade vertical é maior quando comparada à saída do atletismo e, desta forma, o ângulo de entrada na água na saída de agarre também é maior quando comparado a saída do atletismo. Esse aspecto acaba aumentando o tempo na fase subaquática e consequentemente o tempo da prova (VAGHetTi, Berneira \& Roesler, 2010).

Analisando as variáveis de performance na saída de bloco, em relação a $\mathrm{d}_{\text {voo }}$, observaramse semelhanças reportadas em outros estudos (Blanksby, Nicholson \& Elliot, 2002; Hubert, 2005; Miller, Allen \& Pein 2003; Ruschel et al., 2007), entre 2,90 e 3,30 m. Somente no trabalho de VAGHETti, Berneira e Roesler (2010), os nadadores apresentaram d de $4,10 \mathrm{~m}$ após a saída de bloco, bastante superiores aos achados deste estudo.

Quanto ao $\mathrm{t}_{\text {voo }}$, os nadadores participantes deste estudo alcançaram em média $0,40 \mathrm{~s}$ de voo durante a saída de bloco. Alguns estudos (JorGić et al., 2010; Ruschel et al., 2007; VILAS-BOAS et al., 2001) encontraram valores entre 0,33 e 0,34 s. Já Cipolli (2005) verificou valores de 0,27 s e Miller, Allen e Pein (2003) reportaram um tempo de voo de 0,25 s para a saída de agarre, todos menores do que os nadadores deste estudo. Acredita-se que a maior média desta variável possa ser explicada possivelmente por uma maior altura atingida na fase aérea, o que implicaria em maior tempo de voo. Além disso, outras variáveis, como a velocidade da decolagem, que não foi avaliada neste estudo pode ter contribuído para essa diferença.

Por fim, não foram observadas correlações significativas entre os indicadores neuromusculares obtidos no SJ (altura, potência e pico de velocidade) e as variáveis de desempenho na saída de bloco $\left(\mathrm{d}_{\text {voo }}\right.$ e $\mathrm{t}_{\text {voo }}$ ). Tais achados divergiram de SEIFERT et al. (2010), os quais encontraram correlação da altura e da potência no "counter movement jump" (salto vertical que utiliza um contra-movimento) com o ângulo de saída de bloco $(\mathrm{r}=0,39$ e $\mathrm{r}=0,40$, respectivamente) e com o tempo de voo $(r=0,44$ e 0,44 , respectivamente) em nadadores velocistas. Não foram observados estudos com nadadores da mesma especialidade (meio-fundistas) que possibilitassem uma comparação mais aprofundada dos resultados obtidos, apesar da destacada importância da saída para as diferentes especialidades (HUBERT, 2005), e que, segundo MAGLISCHO (2003), resulta na melhora de $0,1 \mathrm{~s}$ em média nos tempos de prova.

Considera-se que a potência de membros inferiores é uma qualidade física importante para a saída de bloco, conforme apontando também em outras investigaçôes (BOCALINI et al., 2007; BREED 
\& Young, 2003; West et al., 2010). No entanto, ainda não existem evidências que apontem um teste que melhor avalie a potência de forma específica e que consiga representar o desempenho na saída de bloco. Dentre os testes existentes, os saltos verticais são amplamente utilizados para avaliar potência em diferentes modalidades esportivas (MARKOVIC, Dizdar, Jukic \& Cardinale, 2004).

Por outro lado, a falta de sensibilidade dos saltos verticais para predizer o desempenho em tarefas específicas, como a saída de bloco na natação, parece ser uma das limitações desse tipo de avaliação (De La Fuente, García \& Arellano, 2003). Isso pode ser evidenciado no estudo de BREED e YounG (2003), os quais reportaram que após oito semanas de treinamento de força, os sujeitos apresentaram ganho no teste de salto vertical, mas este ganho não foi transferido diretamente para o desempenho da saída do bloco. Esta dificuldade em estabelecer relaçōes entre componentes neuromusculares e o desempenho da saída de certa forma continua, e de igual forma não foram encontradas relações significativas neste estudo. Apesar disso, acredita-se que preparadores físicos devem enfatizar em seus treinamentos estímulos de potência
(BOCALINI et al., 2007; WeST et al., 2010), que podem ser que sejam contemplados com sessōes de "sprints", multi-saltos, pliometria ou a combinação destes.

Ainda, os resultados deste estudo podem servir para técnicos de natação, sobretudo aqueles que trabalham com nível de rendimento intermediário, para que enfatizem uma técnica correta de saída de bloco, considerando principalmente o ângulo de saída de bloco e a altura máxima de quadril, utilizando filmagens para detectar estas variáveis. Isto vai ao encontro ao destacado por MAKARENKO (2001), que recomenda que as variantes nos estilos de saída devem ser testadas para decidir qual delas corresponde mais às possibilidades individuais de cada nadador. Pereira (2009) acrescenta que a necessidade dos nadadores de melhorar continuamente sugere o uso de avanços técnico-científicos na investigação das técnicas, pela importância que representam na performance. Assim, mesmo não destacando determinados valores como ótimos dentro das variáveis da saída, a quantificação e relação com o desempenho final devem ser observados, visando a técnica correta e o melhor desempenho para cada nadador.

\section{Conclusões}

Com base nos resultados pode-se concluir que o desempenho inicial da saída de bloco na natação está relacionado diretamente com o ângulo de saída do bloco, sendo que menores ângulos de saída tendem a resultar numa maior distância e menor o tempo de voo, representando um melhor desempenho. Outra relação observada foi entre o tempo de voo e a altura máxima da articulação do quadril na fase área da saída, sendo que quanto menor altura, menor é o tempo que ele permanecerá no ar. A potência muscular (componentes neuromusculares) não apresentou relação com o desempenho dos nadadores investigados, no entanto, esse assunto requer maiores investigações que analisem a transferência dessa qualidade física para a performance da saída de bloco.

\section{Abstract}

Kinematical and neuromuscular aspects related to performance during the swimming start

The aim of this study was to investigate the association between the performance of the start off the swimming block with kinematical and neuromuscular parameters (flight distance and flight time). Ten male swimmers $(26.4 \pm 6.6$ years, $76.4 \pm 6.6 \mathrm{~kg}, 1.81 \pm 0.07 \mathrm{~m})$ participated in the study. A video camera (30 $\mathrm{Hz}$ ) was used to obtain the kinematic variables: flight distance, flight time, maximum height, start angle and entry angle. Muscle power was assessed via the performance of a Squat Jump on a force plate. Jump height, power and peak of velocity were analyzed. Pearson correlation was used to correlate kinematic and neuromuscular variables with starting performance. The level of significance was set at $5 \%$. A significant correlation between the starting angle and both the flight distance $(r=-0.59)$ and the flight time $(r=0.88)$ 
were found; also, a positive correlation was found between maximum height and flight time $(r=0.59)$. No significant correlation between swimming start performance and neuromuscular parameters $(p>0.05)$ were found. We can conclude that performance in the swimming start was related with the start angle and maximum height. Muscle power did not affect swimming start performance in this study.

UNITERMS: Swimming; Muscle strength; Kinematics.

\section{Referências}

ACHE DIAS, J.; DAL PUPO, J.; REIS, D.C.; MORO, A.R.P.; SANTOS, S.G.; BORGES JUNIOR, N.G. Validity of two methods for estimation of vertical jump height. Journal of Strength and Conditioning Research, Champaign, v.25, n.7, p.2034-9, 2011.

ARAUJO, L.; PEREIRA, S.; GATTI, R.; FREITAS, E.; JACOMEL, G.; ROESLER, H.; VILAS-BOAS, J. Analysis of the lateral push-off in the freestyle flip turn. Journal of Sports Science, London, v.28, n.11, p.1175-81, 2010.

BLANKSBY, B.; NICHOLSON, L.; ELLIOTT, B. Biomechanical analysis of the grab, track and handle swimming starts: an intervention study. Sports Biomechanics, Adingdon, v.1, n.1, p.11-24, 2002.

BLANKSBY, B.; SIMPSON, J.; ELLIOT, B.; MCELROY, K. Biomechanical factors influencing breastroke turns by agegroup swimmers. Journal of Applied Biomechanics, Champaign, v.14, n.2, p.180-9, 1998.

BOCALINI, D.S.; ANDRADE, R.M.P.; UEZU, P.T.; SANTOS, R.N.; NAKAMOTO, F.P. O treinamento pliométrico melhora o desempenho da saída de bloco de nadadores. Revista Brasileira de Educação Física, Esporte, Lazer e Dança, Santo André, v.2, n.1, p.1-8, 2007.

BREED, R.V.P.; YOUNG, W.B. The effect of resistence training programme on the grab, track and swing starts in swimming. Journal of Sports Science, London, v.21, n.3, p.213-20, 2003.

BURKETT, B.; MELLIFONT, R.; MASON, B. The influence of swimming start components for selected Olympic and Paralympic swimmers. Journal of Applied Biomechanics, Champaign, v.26, n.2, p.134-41, 2010.

CIPOLLI, E.A.C. Análise cinemática da saída na natação através de um sistema bi-dimensional. 2005. 62 f. Dissertação (Mestrado em Engenharia Mecânica) - Faculdade de Engenharia de Guaratinguetá, Universidade Estadual Paulista, Guaratinguetá, 2005. COSSOR, J.; MASON, B. Swim start performances at the Sydney 2000 Olympic Games. In: INTERNATIONAL SYMPOSIUM ON BIOMECHANICS IN SPORTS, 19., 2001, San Francisco. Proceedings... San Francisco: University of San Francisco, 2001, p.70-4.

DAL PUPO, J.; DETANICO, D. SANTOS, S.G. Parâmetros cinéticos determinantes do desempenho nos saltos verticais. Revista Brasileira de Cineantropometria e Desempenho Humano, Florianópolis, 2011 (in press).

DALY, D.; MALONE, L.; SMITH, D.; VANLANDEWIJCK, Y.; STEADWARD, R. The contribution of starting, turning, and finishing to total race performance in male Paralympic swimmers. Adapted Physical Activity Quarterly, Champaign, v.18, n.3, p.316-33, 2001.

DE LA FUENTE, B.; GARCÍA, F.; ARELLANO, R. Are the forces in the vertical countermovement jump related to the forces applied during the swimming start? In: BIOMECHANICS AND MEDICINE IN SWIMMING, 9., 2003, Saint Etienne. Proceedings... Saint Etienne: University of Saint Etienne, 2003, p.207-12.

GARRIDO, N.; MARINHO, D.A.; REIS, V.M.; VAN DEN TILLAAR, R.; COSTA, A.M.; SILVA, A.J.; MARQUES, M.C. Does combined dry land strength and aerobic training inhibit performance of young competitive swimmers? Journal of Sports Science and Medicine, Belconnen, v.9, n.2, p.300-10, 2010.

GUIMARĀES, A.; HAY, J. A mechanical analysis of the grab starting technique in swimming. International Journal of Sport Biomechanics, Champaign, v.1, n.1, p.25-35, 1985.

HALJAND, R. Technicalpreparation of swimming starts, turns and strokes. Estonia: Tallin University of Education Sciences, 1998.

HUBERT, M. Estudo do procedimento para coleta de dados biomecânicos das saídas no nado crawl. 2005.86 f. Dissertação (Mestrado em Ciências do Movimento Humano) - Curso de Pós-Graduação em Educação Física, Universidade do Estado de Santa Catarina, Florianópolis, 2005.

JORGIC, B.; PULETIC, M.; STANKOVIC, R.; OKICIC, T.; BUBANJ, S.; BUBANJ, R. The kinematic analysis of the grab and track start in swimming. Physical Education and Sport, Nis, v.8, n.1, p.31-6, 2010. 
LEWIS, S. Comparison of five swimming starting techniques. Swimming Technique, North Hollywood, v.15, n.4, p.124-8, 1980.

MAGLISCHO, E.W. Swimming even faster. Mountain View: Mayfield, 2003.

MAKARENKO, L.P. Natação: seleção de talentos e iniciação desportiva. Porto Alegre: Artmed, 2001.

MARKOVIC, G.D; DIZDAR, I.; JUKIC, M.; CARDINALE, M. Reliability and factorial validity of squat and countermovement jump tests. Journal of Strength and Conditioning Research, Champaing, v.18, n.3, p.551-5, 2004.

McLEAN, S.P.; HOLTHE, M.J.; VINT, P.F.; BECKETT, K.D.; HINRICHS, R.N. Addition of an approach to a swimming relay start. Journal of Applied Biomechanics, Champaign, v.16, n.4, p.342-55, 2000.

MILLER, M.; ALLEN, D.; PEIN, R. A kinetic and kinematic comparison of the grab and track starts in swimming. In: WORLD SYMPOSIUM ON BIOMECHANICS AND MEDICINE IN SWIMMING, 9., 2003, Saint Etienne. Proceedings.... Saint Etienne: University of Saint-Etienne, 2003.

PEREIRA, S.M. Estudo biomecânico de quatro técnicas de virada com rolamento em provas de nado livre. 2009. 350 f. Tese (Doutorado em Ciências do Desporto) - Curso de Pós-Graduação em Ciências do Desporto, Universidade do Porto, Porto, 2009.

RUSCHEL, C.; ARAUJO, L.G.; PEREIRA, S.M.; ROESLER, H. Kinematical analysis of the swimming start: block, flight and underwater phases. In: INTERNATIONAL SYMPOSIUM ON BIOMECHANICS IN SPORTS, 25., 2007, Ouro Preto. Anais... Ouro Preto: , 2007. p.385-88.

SEIFERT, L.; VANTORRE, J.; CHOLLET, D. Biomechanical analysis of the breaststroke start. International Journal of Sports Medicine, Stuttgart, v.28, n.11, p.970-6, 2007.

SEIFERT, L.; VANTORRE, J.; LEMAITRE, F.; CHOLLET, D.; TOUSSAINT, H.M.; VILAS-BOAS, J.P. Different profiles of the aerial start phase in front crawl. Journal of Strength \& Conditioning Research, Champaign, v.24, n.2, p.507-16, 2010.

TAKEDA, T.; ICHIKAWA, H.; TAKAGI, H.; TSUBAKIMOTO, S. Do differences in initial speed persist to the stroke phase in front-crawl swimming? Journal of Sports Sciences, London, v.27, n.13, p.1449-54, 2009.

TAKEDA, T.; NOMURA, T. What are the differences between grab and track start? Revista Portuguesa de Ciências do Desporto, Porto, v.6, n.2, p.102-5, 2006. Suplemento 2.

VAGHETTI, C.A.O.; BERNEIRA, J.O.; ROESLER, H. Análise cinemática da saída de agarre na natação. Lecturas, Educación Física y Deportes, Buenos Aires, ano 14, n.140, 2010. Disponível em: <http://www.efdeportes.com/efd140/ analise-cinematica-da-saida-de-agarre-na-natacao.htm>.

VANTORRE, J.; SEIFERT, L.; FERNANDES, R.J.; VILAS BOAS, J.P.; CHOLLET, D. Kinematical profiling of the front crawl start. International Journal of Sports Medicine, Stuttgart, v.31, n.1, p.16-21, 2010a.

. Comparison of grab start between elite and trained swimmers. International Journal of Sports Medicine, Stuttgart, v.31, n.12, p.887-93, 2010b.

VILAS-BOAS, J.P.; CRUZ, M.J.; SOUSA, F.; CONCEIÇÃO, F.; CARVALHO, J.M. Integrated kinematical and dynamics analysis of two track-start techniques. In: INTERNATIONAL SYMPOSIUM ON BIOMECHANICS IN SPORTS, 23. 2001, Hong Kong. Proceedings... Hong Kong: University of Hong Kong, 2001. p.1-5.

WELCHER, R.L.; HINRICHS, R.N.; GEORGE, T.R. Front- or rear-weighted track start or grab start: which is the best for female swimmers? Sports Biomechanics, Abingdon, v.7, n.1, p.100-13, 2008.

WEST, D.J.; OWEN, N.J.; CUNNINGHAM, D.J.; COOK, C.J.; KILDUFF, L.P. Strength and power predictors of swimming starts in international sprint swimmers. Journal of Strength \& Conditioning Research, Champaign, v.25, n.4, p.950-5, 2010.

ENDEREÇO

Daniele Detanico

R. Prof. J osé Brasilício de Souza, 99 - apto. 302 - Trindade 88036-530 - Florianópolis - SC - BRASIL e-mail: danieledetanico@gmail.com
Recebido para publicação: 28/03/2011

1. Revisão: 06/ 06/ 2011

2․ Revisão: 07/ 07/ 2011

Aceito: 26/08/2011

566 • Rev. bras. Educ. Fís. Esporte, São Paulo, v.25, n.4, p.559-66, out./ dez. 2011 